\title{
Electronegativity Equalization and the Electronic Structure of Polyhedral Clusters of Main-Group Atoms
}

\author{
LAWRENCE L. LOHR, JR. \\ Department of Chemistry, University of Michigan, Ann Arbor, Michigan 48109, U.S.A.
}

\begin{abstract}
The concept of the equalization of atomic electronegativities accompanying molecule formation is applied to a study of the electronic structure of polyhedral clusters of main-group atoms such as $\mathrm{Ge}, \mathrm{Sn}, \mathrm{Pb}, \mathrm{Tl}$, and $\mathrm{Bi}$. Emphasis is placed upon charged clusters such as $\mathrm{Sn}_{9-x} \mathrm{~Pb}_{x}^{4-}(x=0 \rightarrow 9)$, $\mathrm{Sn}_{9-x} \mathrm{Ge}_{x}^{4-}, \mathrm{Sn}_{8-x} \mathrm{~Pb}_{x} \mathrm{Tl}^{5-}, \mathrm{Sn}_{2} \mathrm{Bi}_{2}^{2-}, \mathrm{SnTe}_{4}^{4-}$, etc. The role of the relativistic spin-orbit splitting of an $n p$ shell into $n p_{1 / 2}$ and $n p_{3 / 2}$ subshells in modifying atomic and hence molecular electronegativities is discussed. Correlations are made between calculated charge distributions and observed ${ }^{199} \mathrm{Sn}$ NMR chemical shifts for clusters of a given size and charge. It is concluded that a useful picture of charge distributions in these clusters may be obtained from electronegativity equalization considerations.
\end{abstract}

\section{Introduction}

In a recent paper [1] we presented the results of our studies of the electronic structure of charged polyhedral clusters of $\mathrm{Ge}, \mathrm{Sn}, \mathrm{Pb}$, and $\mathrm{Bi}$ atoms using our relativistically parametrized extended Hückel (REX) method [2]. The clusters considered included $\mathrm{Ge}_{9}^{4-}, \mathrm{Ge}_{9}^{2-}, \mathrm{Sn}_{4}^{2-}, \mathrm{Sn}_{5}^{2-}, \mathrm{Sn}_{9}^{4-}, \mathrm{Pb}_{5}^{2-}, \mathrm{Pb}_{9}^{4-}, \mathrm{Bi}_{9}^{5+}$, the heteronuclear clusters $\mathrm{PbSn}_{4}^{2-}, \mathrm{SnGe}_{8}^{4-}, \mathrm{GeSn}_{8}^{4-}, \mathrm{PbSn}_{8}^{4-}, \mathrm{TlSn}_{8}^{5-}$, and the exopolyhedral cluster $\mathrm{CH}_{3} \mathrm{~Pb}_{9}^{3-}$. The study included a discussion of the limited charge dependence of atomic electronegativity as implied by our REX parametrization. The role of relativity in modifying the charge dependence was briefly discussed. In the present study we shall explore in some depth the relationships between relativity and the charge dependence of electronegativity. Again our focus will be upon clusters of main group elements, although the approach will be empirical rather than semi-empirical.

\section{Variation of Electronegativity with Charge}

The electronegativity $\chi$ of a chemical species, atomic or molecular, has been defined [3] as the negative of the electronic chemical potential $\mu$, the latter being the derivative of the energy $E$ with respect to the number of electrons $N$, so that

$$
\chi(N)=-\mu(N)=-(\partial E / \partial N)_{v},
$$

where the subscript $v$ denotes the constancy of the potential. It should be emphasized that $N$ for an isolated species is a discrete rather than the continuous variable implied by Eq. (1), although in applications to atoms or fragments which are a part of an extended system (molecule) it is taken to be continuous. 
The chemical potential $\mu$ is the same as that appearing in the Fermi-Dirac distribution for the electrons,

$$
N_{i}=1 /\left[e^{\left(\epsilon_{i}-\mu\right) / k T}+1\right]
$$

where $N_{i}$ is the number of fermions $\left(0 \leq N_{i} \leq 1\right)$ in a single-particle state with energy $\epsilon_{i}$ and $k$ is the Boltzmann constant. The requirement that the sum of the $N_{i}$ be constant may be used to determine $\mu$, which is typically a slowly varying function of $T$.

Rather than assuming the total energy of a species to be a quadratic function of its total number of electrons, as done by Iczkowski and Margrave [4] as well as by others, we first consider a more general polynomial representation,

$$
E(N)=\sum_{i=1}^{j_{\max }} a_{i} N^{i}
$$

where both $E$ and $N$ are taken to be zero for a neutral species, so that $N$ represents the electron excess (positive for an anion, negative for a cation). Thus $x$ becomes

$$
\chi(N)=-\sum_{j=i}^{i_{\max }} j a_{j} N^{i-i} .
$$

In Figs. 1 and 2 we show quadratic polynomials fitted exactly to the energies of $\mathrm{A}^{+}(N=-1), \mathrm{A}^{0}(N=0)$, and $\mathrm{A}^{-}(N=+1)$ for $\mathrm{A}=\mathrm{As}$ and $\mathrm{A}=\mathrm{Bi}$, respectively.

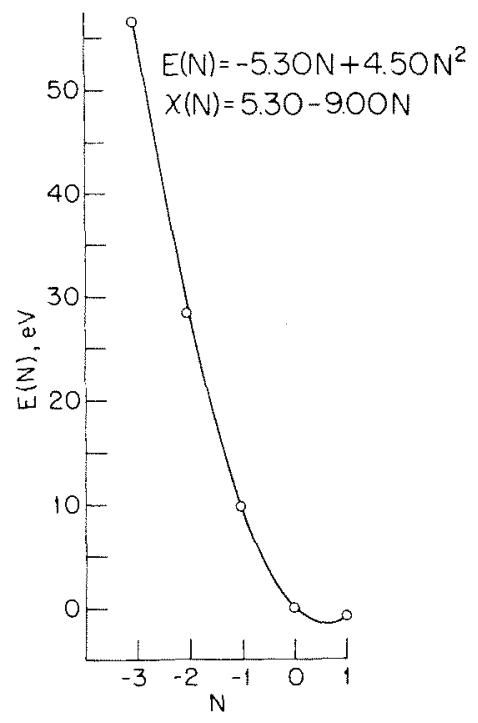

Figure 1. Quadratic representation of the energy in $\mathrm{eV}$ of atomic As as function of number of electrons $N$. Curve fitted to energies of $\mathrm{As}^{+}, \mathrm{As}^{0}$, and $\mathrm{As}^{-1}(N=-1,0,+1$, respectively). Circles denote these energies as well as those for $\mathrm{As}^{3+}(N=-3)$ and $\mathrm{As}^{2+}(N=-2)$. 


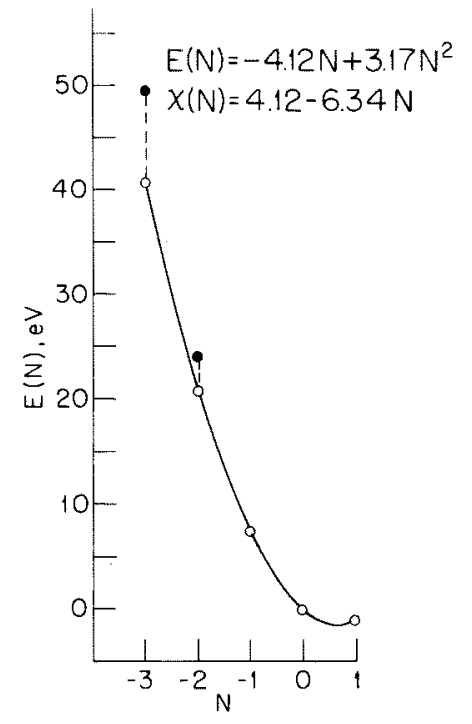

Figure 2. Quadratic representation of the energy in $\mathrm{eV}$ of atomic $\mathrm{Bi}$ as a function of $N$. Curve fitted as in Figure 1 . Solid points for $N=-3$ and $N=-2$ denote the observed energies of $\mathrm{Bi}^{3+}$ and $\mathrm{Bi}^{2+}$, which differ significantly from the corresponding open points on the curve.

The data used are the ionization energies [5], (IE), of 9.81 and $7.29 \mathrm{eV}$ and the electron affinities of 0.80 and $0.947 \mathrm{eV}$ for $\mathrm{As}$ [6] and $\mathrm{Bi}$ [7], respectively. We note that while the experimental energies of $\mathrm{As}^{2+}(28.44 \mathrm{eV})$ and $\mathrm{As}^{3+}(56.79 \mathrm{eV})$ fall very close to the values of 28.60 and $56.40 \mathrm{eV}$ given by the quadratic form, the corresponding experimental values for $\mathrm{Bi}^{2+}(23.98 \mathrm{eV})$ and $\mathrm{Bi}^{3+}(49.54)$ lie significantly above the values of 20.92 and $40.89 \mathrm{eV}$ given by the quadratic form. This is a relativistic effect, in that $\mathrm{Bi}$ is characterized by a $6 p_{1 / 2}, 6 p_{3 / 2}$ subshell structure, so a quadratic fitting to the energies of $\mathrm{Bi}^{+}, \mathrm{Bi}^{\circ}$, and $\mathrm{Bi}^{-}$, differing in the occupancy of the destabilized $6 \mathrm{p}_{3 / 2}$ subshell, underestimates the energies of $\mathrm{Bi}^{2+}$ and $\mathrm{Bi}^{3+}$, which involve removal of electrons from the spin-orbit stabilized $6 p_{1 / 2}$ subshell. The corresponding effect in the $4 p$ shell of As is negligible by comparison. If a cubic term in $N$ is included in Eq. (1), and fitted using the energy of $A^{2+}$ in addition to that of $\mathrm{A}^{+}, \mathrm{A}^{0}$, and $\mathrm{A}^{-}$, the cubic coefficients for $\mathrm{As}$ and $\mathrm{Bi}$ are +0.03 and $-0.51 \mathrm{eV}$, respectively, the size of the coefficient for $\mathrm{Bi}$ reflecting the inadequacy of a quadratic form over a large range of $N$. For small ranges of $N$ a quadratic form should be adequate even for a heavy element, provided that the form is fitted to points in the range for which it is to be used. Thus for obtaining the variation of the energy of $\mathrm{Bi}$ with respect to the $6 p_{1 / 2}$ occupancy the energies of $\mathrm{Bi}^{3+}, \mathrm{Bi}^{2+}$ and $\mathrm{Bi}^{+}$should provide a good description, namely a polynomial with coefficients $a_{0}$ (constant) $=-0.18, a_{1}=$ -3.09 , and $a_{2}=3.25 \mathrm{eV}$. This form, however, assigns values of -0.18 and $-0.02 \mathrm{eV}$ to $\mathrm{Bi}^{\circ}$ and $\mathrm{Bi}^{-}$, respectively, and so should not be used for these species involving $6 p_{3 / 2}$ occupancy. 
In Table I we present values of the linear and quadratic coefficients $a_{1}$ and $a_{2}$ as obtained from IE and EA data [5-8] for a number of elements including those of interest in this study. All values are thus fitted to free-atom energies for $\mathrm{A}^{+}, \mathrm{A}^{0}$, and $\mathrm{A}^{-}$, whose charges span the range of the average charges per atom $(q / n)$ in the clusters of interest, such as $\operatorname{Sn}_{4}^{2-}(-1 / 2), \mathrm{Sn}_{9-x} \mathrm{~Pb}_{x}^{4-}(-4 / 9)$, $\mathrm{Bi}_{9}^{5+}(+5 / 9)$, etc.

TABLE 1. Linear and quadratic energy coefficients."

\begin{tabular}{lllllr}
\hline Element & \multicolumn{1}{c}{$\mathrm{IE}^{\mathrm{b}}$} & \multicolumn{1}{c}{$\mathrm{EA}^{\mathrm{c}}$} & $-a_{1}{ }^{\mathrm{d}}$ & $a_{2}{ }^{\mathrm{e}}$ & IE(av) $^{\mathrm{f}}$ \\
\hline $\mathrm{Ga}$ & 5.999 & $0.30(15)$ & 3.15 & 2.85 & 5.93 \\
$\mathrm{Ge}$ & 7.899 & $1.2(1)$ & 4.55 & 3.35 & 7.53 \\
$\mathrm{As}$ & 9.81 & $0.80(5)$ & 5.30 & 4.50 & 9.18 \\
$\mathrm{Se}$ & 9.752 & $2.0206(3)$ & 5.89 & 3.86 & 10.82 \\
$\mathrm{In}$ & 5.786 & $0.30(15)$ & 3.04 & 2.74 & 5.60 \\
$\mathrm{Sn}$ & 7.334 & $1.25(10)$ & 4.30 & 3.05 & 7.01 \\
$\mathrm{Sb}$ & 8.641 & $1.05(5)$ & 4.84 & 3.80 & 8.44 \\
$\mathrm{Te}$ & 9.009 & $1.9708(3)$ & 5.49 & 3.52 & 9.78 \\
$\mathrm{Tl}$ & 6.108 & $0.3(2)$ & 3.20 & 2.90 & 5.46 \\
$\mathrm{~Pb}$ & 7.416 & $0.365(8)^{\mathrm{g}}$ & 3.89 & 3.53 & 6.81 \\
$\mathrm{Bi}$ & 7.289 & $0.947(10)^{\mathrm{p}}$ & 4.12 & 3.17 & 8.14 \\
$\mathrm{Po}$ & 8.42 & $1.9(3)$ & 5.16 & 3.26 & 6.70 \\
$\mathrm{Pt}$ & 9.0 & $2.128(2)$ & 5.6 & 3.4 & - \\
\hline \hline
\end{tabular}

${ }^{a}$ All energies in $\mathrm{eV}$.

${ }^{\mathrm{h}}$ Data from Ref. [5].

${ }^{\mathrm{c}}$ Data from Ref. [6] except for $\mathrm{B}, \mathrm{Al}, \mathrm{Pb}$, and $\mathrm{Bi}$ which are from Ref. [7]. Uncertainties in the last figure given in parentheses.

${ }^{d} a_{1} \equiv-(\mathrm{IE}+\mathrm{EA}) / 2$.

$\mathrm{e} a_{2}=(\mathrm{IE}-\mathrm{EA}) / 2$.

f Average-of-configuration IEs from Ref. [8].

${ }^{g}$ Data from Ref. [7].

\section{Electronegativity Equalization}

The concept that if two or more species of generally different electronegativity $x$ join to form a composite species there results a common new electronegativity has been expressed many times [3,9-11] following the ideas of Sanderson [12]. The feature that all parts of the composite species have the same electronegativity corresponds [3] to spatial uniformity of the electronic chemical potential $\mu$. A simple model for the electronegativity of molecules is that based on the equalization of charge-dependent free-atom electronegativities. Ray et al. have applied this mode [10] to a large number of diatomic and polyatomic molecules, while Reed [11] has discussed its limitations. The model in its usual form ignores homopolar bonding effects, so that $\mathrm{H}_{2}$ and $\mathrm{H}$ would be assigned the same $\mu$ and hence $\chi$. However, Reed has pointed out that if the effects of bonding energies are small compared to the total valence electron energy, then the equalization 
of atomic electronegativities corresponds approximately to the minimization of molecular energy, thus justifying the use of the model. In this paper we present its application to a number of mixed main-group cluster molecules, both neutral and charged.

Consider a molecule consisting of $n_{1}$ atoms (or groups) of type $1, n_{2}$ of type 2 , etc. We assume that the molecular energy $E$ is additive,

$$
\sum_{i} n_{i} E_{i}\left(N_{i}\right)=E,
$$

where the energy $E_{i}$ of atom $i$ is some function of the number of electrons $N_{i}$ on that atom and the summation is over atom types. The $\left\{N_{i}\right\}$ are taken to be zero for neutral atoms, as is the molecular total $N$, given by

$$
\sum_{i} n_{i} N_{i}=N
$$

As in the previous section we take the $\left\{N_{i}\right\}$ to be continuous variables, so that minimization of Eq. (5) subject to constraint Eq. (6) by the Lagrange method is equivalent to minimizing $E^{\prime} \equiv E-\mu N$, leading to the set of equations

$$
\partial E^{\prime} / \partial N_{i}=\partial E_{i} / \partial N_{i}-\mu=0 .
$$

If each $E_{i}$ has the polynomial form of Eq (1) but is limited to terms no higher than quadratic, then

$$
N_{i}=\left(\mu-a_{1}^{i} / 2 a_{2}^{i},\right.
$$

where $a_{1}^{i}$ and $a_{2}^{i}$ are the linear and quadratic coefficients of $N_{i}$ in $\mathrm{Eq}(1)$ for atom $i$. Using Eq. (6) one obtains

$$
\mu=\frac{N+\sum_{i}\left(n_{i} a_{1}^{i} / 2 a_{2}^{i}\right)}{\sum_{i}\left(n_{i} / 2 a_{2}^{i}\right)} .
$$

Thus $\mu$ (or $-X$ ) may be calculated from the atomic parameters $\left\{a_{1}^{i}\right\}$ and $\left\{a_{2}^{i}\right\}$, the numbers $\left\{n_{i}\right\}$, and the molecular charge $q=-N$. Values of the $\left\{N_{i}\right\}$ and $E$ are then readily obtained using Eqs. (8) and (5). We note that the energy $E_{i}$ of each isolated atom is a minimum for $N_{i}=N_{i}^{0}=-a_{1}^{i} / 2 a_{2}^{i}$; these $\left\{N_{i}^{0}\right\}$ also minimize $E$ as a function of $N$ given the additivity in $\mathrm{Eq}$. (5). Thus $\mu$ may be written as

$$
\mu=\frac{\sum_{i} n_{i}\left(N_{i}-N_{i}^{0}\right)}{\sum_{i}\left(n_{i} / 2 a_{2}^{i}\right)},
$$

where $N_{i}-N_{i}^{0}$ is the difference between the number of electrons on atom $i$ which minimizes the molecular $E$ for fixed $N$ and the number which minimizes it for variable $N$. 
Although the parameter $\mu$ follows from the constraint of constant $N$ in Eq. (6), its value is readily related to the variation of $E$ with respect to $N$. We first note that $E_{i}$, within the quadratic approximation, may be expressed using Eq. (8) as

$$
E_{i}=\left[\mu^{2}-\left(a_{1}^{i}\right)^{2}\right] / 4 a_{2}^{i},
$$

where $\mu$ is a linear function of $N$ given by Eq. (9). Substitution of Eq. (11) into Eq. (5) yields $E(\mu(N)]$, from which it follows that

$$
\begin{aligned}
& {[E(N+1)-E(N)]=(d E / d N)_{N+\frac{1}{2}}=\mu\left(N+\frac{1}{2}\right),} \\
& {[E(N+1)-E(N-1)] / 2=(d E / d N)_{N}=\mu(N),} \\
& {[E(N)-E(N-1)]=(d E / d N)_{N-\frac{1}{2}}=\mu\left(N-\frac{1}{2}\right) .}
\end{aligned}
$$

The relationships in Eq. (12) simply reflect the fact that if the energy $E_{i}$ of the $i$ th atom (or group) is a quadratic function of $N_{i}$ so that the Mulliken approximation to $\mu_{i}$ and hence $\chi_{i}$ is exact for that atom, and if the molecular energy $E$ is additive as in Eq. (5), then the Mulliken approximation is also exact for the molecular $\mu$ and hence $\chi$. It follows that a one-electron energy difference may be obtained from a knowledge of $\mu\left(N+\frac{1}{2}\right)$ by Eqs. (12a) or (12c), while a two-electron energy difference may be obtained from a knowledge of $\mu(N)$ by Eq. (12b).

The preceding relationships are similar to those of Ray et al. [10] in their charge transfer model of electronegativity equalization. Our formulation is for arbitrary $N$, not just zero. However there is an added uncertainty as to the significance of the model when it is applied to a charged molecular species. Some examples of the effects of counterions are given in the next section. Finally, we note the particularly simple form for the $\left\{N_{i}\right\}$ as given by Eq. (8) in terms of $\mu$ from Eq. (9) and the constancy of $d \mu / d N$ with a value from Eq. (9) of $1 /\left[\sum_{i}\left(n_{i} / 2 a_{2}^{i}\right)\right]$.

\section{Applications to Main-Group Clusters}

We now consider the application of the electronegativity equalization model to various clusters of main-group elements. Many of these clusters contain Sn, as ${ }^{119} \mathrm{Sn}$ NMR has proven [13-15] to be particularly valuable in the identification of solution species in solvents such as ethylenediamine (en). Although atomic electronegativities might be taken from either nonrelativistic [16] or relativistic [17] $X \alpha$ calculations, we have chosen instead to use atomic electronegativities taken from the empirical data of Table I. Specifically we have used the average of configuration IEs given in the last column of the table. The use of empirical parameters implicitly incorporates relativistic effects in to the model. For example, the recently reported [7] EA value of $0.365 \mathrm{eV}$ for $\mathrm{Pb}$ is quite small compared to the values for Ge and $\mathrm{Sn}$, reflecting the spin-orbit destabilization of the $6 p_{3 / 2}$ subshell which is unoccupied in $\mathrm{Pb}^{0}$ but occupied in $\mathrm{Pb}^{-}$. As a consequence the electronegativity of $\mathrm{Pb}$ is less than that of $\mathrm{Ge}$ or $\mathrm{Sn}$ for atomic charges near 
$-\frac{1}{2}\left(N=+\frac{1}{2}\right)$, although the customary ordering with $\chi(\mathrm{Pb})>\chi(\mathrm{Sn})$ obtains for positive oxidation states such as +2 .

In Table II we list the computed charges and $\mu$ values for clusters $\mathrm{Ge}_{9-x} \mathrm{Sn}_{x}^{4--}$, $\mathrm{Sn}_{9-x} \mathrm{~Pb}_{x}^{4-}$, and $\mathrm{Pb}_{9-x} \mathrm{Ge}_{x}^{4-}, x=0 \rightarrow 9$. Many of these clusters have been identified [13-15] in solution by NMR and have also been the subject of our semi-empirical

TABLE II. Electronegativity equalization results for $\mathrm{A}_{9-x} B_{x}^{4-}$ clusters.

\begin{tabular}{|c|c|c|c|c|c|c|c|c|c|}
\hline \multirow[b]{2}{*}{$x$} & \multicolumn{3}{|c|}{$\mathrm{A}=\mathrm{Ge}, \mathrm{B}=\mathrm{Sn}$} & \multicolumn{3}{|c|}{$\mathrm{A}=\mathrm{Sn}, \mathrm{B}=\mathrm{Pb}$} & \multicolumn{3}{|c|}{$\mathrm{A}=\mathrm{Pb}, \mathrm{B}=\mathrm{Ge}$} \\
\hline & $q_{\mathrm{A}}$ & $q_{\mathrm{B}}$ & $\mu^{a}$ & $q_{\mathrm{A}}$ & $q_{\mathrm{B}}$ & $\mu^{\text {" }}$ & $q_{\mathrm{A}}$ & $q_{\mathrm{B}}$ & $\mu^{\mathrm{a}}$ \\
\hline 0 & -0.444 & - & -1.552 & -0.44 & - & -1.57 & -0.44 & - & -0.72 \\
\hline 1 & -0.444 & -0.447 & -1.554 & -0.46 & -0.33 & -1.48 & -0.43 & -0.56 & -0.82 \\
\hline 2 & -0.444 & -0.447 & -1.556 & -0.47 & -0.34 & -1.40 & -0.42 & -0.54 & -0.91 \\
\hline 3 & -0.443 & -0.446 & -1.558 & -0.49 & -0.35 & -1.31 & -0.40 & -0.53 & -1.00 \\
\hline 4 & -0.443 & -0.446 & -1.560 & -0.50 & -0.37 & -1.22 & -0.39 & -0.52 & -1.10 \\
\hline 5 & -0.443 & -0.446 & -1.562 & -0.52 & -0.38 & -1.12 & -0.37 & -0.50 & -1.19 \\
\hline 6 & -0.442 & -0.445 & -1.564 & -0.54 & -0.40 & -1.03 & -0.36 & -0.49 & -1.28 \\
\hline 7 & -0.442 & -0.445 & -1.566 & -0.56 & -0.41 & -0.93 & -0.34 & -0.47 & -1.37 \\
\hline 8 & -0.442 & -0.445 & -1.568 & -0.57 & -0.43 & -0.83 & -0.33 & -0.46 & -1.46 \\
\hline 9 & - & -0.444 & -1.570 & - & -0.44 & -0.72 & - & -0.44 & -1.55 \\
\hline
\end{tabular}

${ }^{\text {a }}$ Values in $\mathrm{eV}$ based on average of configuration IEs from Table I.

REX LCAO-MO investigations [1]. Crystal structures have been reported for several of these clusters, with the idealized molecular structures having $[18,19] \mathrm{C}_{4 v}$ symmetry (capped antiprism) for $\mathrm{Ge}_{9}^{4-}$ and $\mathrm{Sn}_{9}^{4-}$, but having [19] $\mathrm{D}_{3 d}$ (tricapped trigonal prism) for the oxidized form $\mathrm{Ge}^{2-9}$. By contrast the clusters $\mathrm{A}_{9-x} \mathrm{~B}_{x}^{4-}$ all appear [13-15] to be fluxional in solution, as indicated by a single ${ }^{119} \mathrm{Sn}$ chemical shift per cluster. The striking feature of the $\mathrm{Ge}_{9-x} \mathrm{Sn}_{x}^{4-}$ results is that the charges and $\mu$ are essentially independent of $x$, reflecting the near equivalence of $\chi(\mathrm{Ge})$ and $\chi(\mathrm{Sn})$ for $N=+4 / 9$, the average excess electron number in these clusters. In fact, the experimental uncertainty of $\pm 0.1 \mathrm{eV}$ in each of the EA values for $\mathrm{Ge}$ and $\mathrm{Sn}$ in Table I does not permit any significance to be attached to the small trends in charges for these clusters. By contrast the results in Table II for the $\mathrm{Sn}_{9-x} \mathrm{~Pb}_{x}^{4-}$ and $\mathrm{Pb}_{9-x} \mathrm{Ge}_{x}^{4-}$ series show significant variations with $x$ which are nearly linear, as shown in Figure 3 in terms of $N(x)=-q$ for each element in the $\mathrm{Sn}-\mathrm{Pb}$ clusters. The inequality $\chi(\mathrm{Sn})>\chi(\mathrm{Pb})$ in this charge range is clearly indicated by the increasingly negative $\mathrm{Sn}$ charge upon $\mathrm{Pb}$ substitution.

In Tables III and IV we list similarly computed charge and $\mu$ values for numerous additional clusters, including the series $\mathrm{Sn}_{9-x} \mathrm{Bi}_{x}^{x-4}$ and the ternary clusters $\mathrm{Sn}_{8-x} \mathrm{~Pb}_{x} \mathrm{Tl}^{q}(q=-5,-3)$. Several clusters of the latter type have been reported $[14,15]$ both from NMR $(q=-5)$ and $x$-ray crystallographic $\left(\mathrm{Sn}_{8} \mathrm{Tl}^{3-}\right.$ and $\mathrm{Sn}_{9} \mathrm{Tl}^{3-}$ ) data [20]. Results for $\mathrm{Sn}_{8-x} \mathrm{~Pb}_{x} \mathrm{Tl}^{5-}$ parallel those for $\mathrm{Sn}_{9-x} \mathrm{~Pb}_{x}^{4-}$. We note from Table III that the $\mathrm{Sn}$-rich members of the $\mathrm{Sn}_{9-x} \mathrm{Bi}_{x}^{x-4}$ series have 


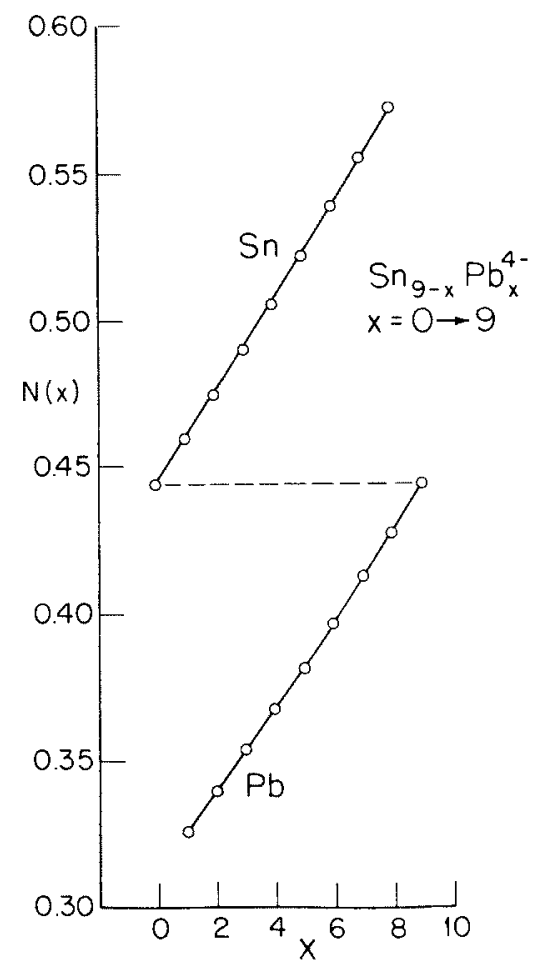

Figure 3. Excess electrons $N(x)$ for $\mathrm{Sn}$ and $\mathrm{Pb}$ in the clusters $\mathrm{Sn}_{9_{-x}} \mathrm{~Pb}_{x}^{4-}, x=0 \rightarrow 9$, as obtained from electronegativity equalization calculations using parameters from

Table I (average of configuration IEs).

nearly equal $\mathrm{Sn}$ and $\mathrm{Bi}$ charges, although $\mathrm{Sn}$ is more positive than $\mathrm{Bi}$ in the $\mathrm{Bi}$-rich clusters. Only $\mathrm{Sn}_{8} \mathrm{Bi}^{3-}$ has been identified in en solution. Other clusters include $\mathrm{Sn}_{2} \mathrm{Bi}_{2}^{2-}$ and $\mathrm{SnTe}_{4}^{4-}$, where the latter species differs from all the others in that it is a coordination polyhedron like $\mathrm{SiO}_{4}^{4-}$.

\section{Relationship to NMR Chemical Shifts}

It is tempting to relate these results to the observed variations in the ${ }^{119} \mathrm{Sn}$ chemical shifts $[13-15,21]$. For the series $\mathrm{Sn}_{9-x} \mathrm{Ge}_{x}^{4-}$ there is a small but nonlinear variation, with an extremum of $+50 \mathrm{ppm}$ for $\mathrm{Sn}_{5} \mathrm{Ge}_{4}^{4-}\left(\mathrm{Na}^{+}\right.$salt in en) relative to $\mathrm{Sn}_{9}^{4-}$. The positive shift corresponds to a shift down field, indicating reduced shielding. The $\delta$ values for $\mathrm{Sn}_{8} \mathrm{Ge}^{4-}$ and $\mathrm{SnGe}_{8}^{4--}$ are surprisingly close, namely +7.0 and $-6.6 \mathrm{ppm}$, respectively. The significant result is that the observed $\delta$ values are essentially independent of $\chi$, a result compatible with our finding of essentially constant computed charges for this series. By contrast the $\mathrm{Sn}_{9_{-x}} \mathrm{~Pb}_{x}^{4-}$ series shows [14] a significant and nearly linear variation of observed $\delta$ values from $-42 \mathrm{ppm}$ for $\mathrm{Sn}_{8} \mathrm{~Pb}^{4-}$ to $-380 \mathrm{ppm}$ for $\mathrm{SnPb}_{8}^{4-}$. The increasingly negative (up field) $\delta$ values indicates increasing shielding of the $\mathrm{Sn}$ nuclei, again a result 
TABLE III. Electronegativity equalization results for various $A_{x} B_{Y}^{8}$ clusters.

\begin{tabular}{llll}
\hline \hline Cluster & $q_{\mathrm{A}}$ & $q_{\mathrm{A}}$ & $\mu$ \\
\hline $\mathrm{Sn}_{8} \mathrm{Sb}^{3-}$ & -0.332 & -0.342 & -2.22 \\
$\mathrm{Sn}_{8} \mathrm{Bi}^{3-}$ & -0.344 & -0.326 & -2.20 \\
$\mathrm{Sn}_{7} \mathrm{Bi}_{2}^{2-}$ & -0.22 & -0.23 & -2.87 \\
$\mathrm{Sn}_{6} \mathrm{Bi}_{3}^{-}$ & -0.10 & -0.14 & -3.56 \\
$\mathrm{Sn}_{5} \mathrm{Bi}_{4}^{0}$ & +0.03 & -0.04 & -4.29 \\
$\mathrm{Sn}_{4} \mathrm{Bi}_{5}^{+}$ & +0.16 & +0.07 & -5.06 \\
$\mathrm{Sn}_{3} \mathrm{Bi}_{6}^{2+}$ & +0.30 & +0.18 & -5.86 \\
$\mathrm{Sn}_{2} \mathrm{Bi}_{7}^{3+}$ & +0.45 & +0.30 & -6.71 \\
$\mathrm{SnBi}_{8}^{4+}$ & +0.60 & +0.42 & -7.60 \\
$\mathrm{Bi}_{9}^{5+}$ & - & +0.55 & -8.54 \\
$\mathrm{Sn}_{2} \mathrm{Bi}_{2}^{2-}$ & -0.52 & -0.48 & -1.12 \\
$\mathrm{Te}_{2} \mathrm{Tl}_{2}^{2-}$ & -0.63 & -0.37 & -0.96 \\
$\mathrm{SnTe}_{4}^{4-}$ & -0.786 & -0.803 & +0.40 \\
$\mathrm{Sn}_{9} \mathrm{Tl}^{3-}$ & -0.32 & -0.12 & -2.28 \\
$\mathrm{Sn}_{9} \mathrm{Tl}^{5-}$ & -0.52 & -0.34 & -1.14 \\
$\mathrm{Sn}_{9} \mathrm{Pt}^{2-}$ & -0.18 & -0.36 & -3.08 \\
$\mathrm{Sn}_{9} \mathrm{Pi}^{4-}$ & -0.38 & -0.53 & -1.91 \\
\hline
\end{tabular}

compatible with our finding of increasingly negative Sn charges as $x$ increases for this series (Table II and Fig. 3).

Simple comparisons of $\delta$ values with computed charges should not be taken too seriously, no matter how accurate the latter might be, even though reasonable correlations of chemical shift data with charges and/or electronegativities have often been made [22]. It is certainly possible that not only the diamagnetic contribution to the nuclear shielding, but also the paramagnetic contribution as related to the variation in the anisotropy in $p$-orbital occupancy, might vary in an essentially linear way with the effective charge on an atom in a molecule.

TABLE IV. Electronegativity equalization results for $\mathrm{Sn}_{8-x} \mathrm{~Pb}_{x} \mathrm{Tl}^{q}$ clusters.

\begin{tabular}{|c|c|c|c|c|c|c|c|c|}
\hline \multirow[b]{2}{*}{$x$} & \multicolumn{4}{|c|}{$q=-5$} & \multicolumn{4}{|c|}{$q=-3$} \\
\hline & $q_{\mathrm{Sn}}$ & $q_{\mathrm{Pb}}$ & $q_{\mathrm{Tl}}$ & $\mu$ & $q_{\mathrm{Sn}}$ & $q_{\mathrm{Pb}}$ & $q_{\mathrm{Tl}}$ & $\mu$ \\
\hline 0 & -0.58 & - & -0.40 & -0.82 & -0.36 & - & -0.15 & -2.08 \\
\hline 1 & -0.59 & -0.44 & -0.42 & -0.72 & -0.37 & -0.24 & -0.17 & -2.00 \\
\hline 2 & -0.61 & -0.46 & -0.44 & -0.63 & -0.38 & -0.26 & -0.18 & -1.92 \\
\hline 3 & -0.62 & -0.47 & -0.45 & -0.53 & -0.40 & -0.27 & -0.20 & -1.84 \\
\hline 4 & -0.64 & -0.49 & -0.47 & -0.43 & -0.41 & -0.28 & -0.22 & -1.76 \\
\hline 5 & -0.66 & -0.50 & -0.49 & -0.33 & -0.43 & -0.30 & -0.23 & -1.67 \\
\hline 6 & -0.68 & -0.52 & -0.51 & -0.22 & -0.44 & -0.31 & -0.25 & -1.58 \\
\hline 7 & -0.70 & -0.54 & -0.54 & -0.12 & -0.46 & -0.32 & -0.27 & -1.49 \\
\hline 8 & - & -0.56 & -0.56 & -0.01 & - & -0.34 & -0.29 & -1.40 \\
\hline
\end{tabular}


Thus as the excess electron population on a Group IV atom increases from 0 in $\mathrm{A}^{0}$ toward +1 in $\mathrm{A}^{-1}$ not only should the diamagnetic shielding increase but also the paramagnetic shielding should decrease, since the $p$-orbital anisotropy should be decreasing as the half-filled shell $p^{3}$ is approached. In a strong spin-orbit coupling limit this description would be changed, as the configuration $p_{1 / 2}^{2} p_{3 / 2}$ is more anisotropic than the spherically symmetric configuration $p_{1 / 2}^{2}$. The effects of spin-orbit coupling on chemical shifts have been noted [23, 24] in a variety of molecules and have been computed [24] via third-order perturbation theory for the halomethanes. Specifically compounds such as $\mathrm{CI}_{4}$ exhibit an anomolously high ${ }^{13} \mathrm{C}$ shielding which cannot be adequately accounted for without a consideration of spin-orbit coupling. Similarly the shift for ${ }^{119} \mathrm{Sn}$ in $\mathrm{SnI}_{4}$ is large [25], namely $-1700 \mathrm{ppm}$ (up field) relative to $\mathrm{Sn}\left(\mathrm{CH}_{3}\right)_{4}$. The isoelectronic species $\mathrm{SnTe}_{4}^{4-}$ exhibits [14] a similar $\delta$ value of $-1828 \mathrm{ppm}\left(\mathrm{Na}^{+}\right.$salt in en) which is $-598 \mathrm{ppm}$ relative to $\mathrm{Sn}_{9}^{4-}$. These shifts, as well as those in the $\mathrm{Sn}_{9-x} \mathrm{~Pb}_{4}^{4-}$ and related series, might very well contain an appreciable spin-orbit contribution. A model [26] for the ${ }^{119} \mathrm{Sn}$ shielding in $\mathrm{SnX}_{4}$ in which the diamagnetic contribution was assumed constant and in which the paramagnetic contribution was taken from spin-rotation constants extracted from relaxation times gave a reasonably satisfactory shift for $\mathrm{SnI}_{4}$ relative to $\mathrm{SnCl}_{4}$. Such an empirical analysis may indeed implicitly incorporate spin-orbit effects.

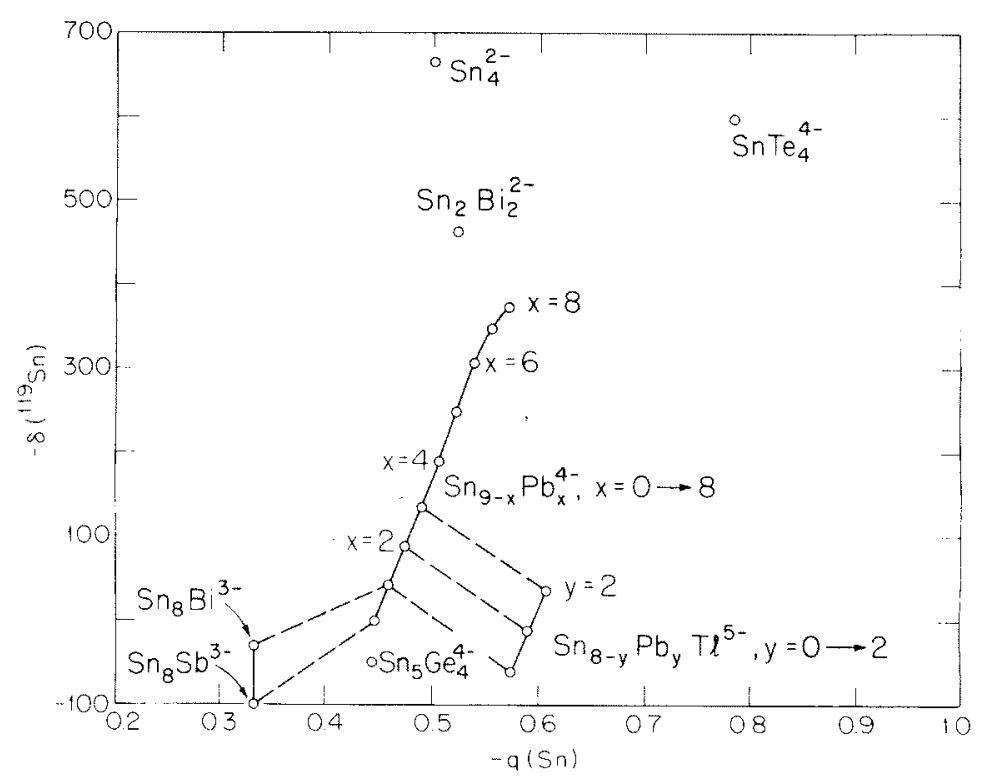

Figure 4. Plot of negative of ${ }^{119} \mathrm{Sn}$ chemical shift $(-\delta)$ vs. Sn electron number $N$ (negative of charge $q$ ) for various clusters. Shifts are for $\mathrm{Na}^{+}$salts in ethylenediamine (en) solution and are relative to that for $\mathrm{Sn}_{9}^{4-}$ taken as zero $(1230.0 \mathrm{ppm}$ upfield from tetramethyltin). Dashed lines connect isoelectronic species, such as $\mathrm{Sn}_{8} \mathrm{Bi}^{3-}$, $\mathrm{Sn}_{\times} \mathrm{Pb}^{+-}, \mathrm{Sn}_{\times} \mathrm{Tl}{ }^{5}$, while solid lines connect species of the same charge differing by replacement of a fifth period element by a sixth period element, such as $\mathrm{Sn}_{9}^{4-}$, $\mathrm{Sn}_{8} \mathrm{~Pb}^{4}, \mathrm{Sn}_{7} \mathrm{~Pb}_{2}^{4-}$, etc. Experimental data from Refs. 13-15. 
In Figure 4 we summarize many of the observed ${ }^{119} \mathrm{Sn} \delta$ values in a plot vs. the Sn charge computed from electronegativity consideration. No simple overall relationship is either found or expected, although there is a rough linear correlation, as previously noted, for clusters of a given size and charge. Our conclusion is that the electronegativity equalization model appears to describe reasonably well the general features of the charge distributions in these clusters, and for this limited purpose is superior to noniterative molecular orbital methods which often yield exaggerated distributions.

\section{Acknowledgements}

The author wishes to thank Mr. W. L. Wilson for many valuable discussions and for permission to quote NMR chemical shift data obtained by him and the late Professor R. W. Rudolph.

\section{Bibliography}

[1] L. L. Lohr, Inorg. Chem. 20, 4229 (1981). [Part V in REX series, with Refs. 2a-d and e comprising Parts I-IV and VI.]

[2] (a) L. L. Lohr and P. Pyykkö, Chem. Phys. Lett. 62, 333 (1979). (b) L. L. Lohr, M. Hotokka, and P. Pyykkö, Int. J. Quantum Chem. 18, 347 (1980). (c) P. Pyykkö and L. L. Lohr, Inorg. Chem. 20, 1950 (1981). (d) P. Pyykkö and L. Wisenfeld, Mol. Phys. 43, 557 (1981). (e) P. Pyykkö, J. Organometallic Chem. 232, 21 (1982). (f) L. Lohr, M. Horokka, and P. Pyykkö, QCPE 12, 387 (1980).

[3] R. G. Parr, R. A. Donnelly, M. Levy, and W. E. Palke, J. Chem. Phys. 68, 3801 (1978).

[4] R. P. Iczkowski and J. L. Margrave, J. Am. Chem. Soc. 83, 3547 (1961).

[5] C. E. Moore, Ionization Potentials and Ionization Limits Derived from the Analyses of Optical Spectra, Nat. Stand. Ref. Data Ser. (Nat. Bur. Stand. (U.S.), 1970), Vol. 34.

[6] H. Hotep and W. C. Lineberger, J. Phys. Chem. Ref. Data 4, 539 (1975).

[7] C. S. Feigerle, R. R. Corderman, and W. C. Lineberger, J. Chem. Phys. 74, 1513 (1981).

[8] C. E. Moore, Atomic Energy Levels as Derived from the Analyses of Optical Spectra (Nat. Bur. Stand. (U.S.) Circular 467), Vol. I, 1949; Vol. II, 1952; Vol. III, 1958.

[9] J. E. Huheey, J. Phys. Chem. 69, 3284 (1965).

[10] N. K. Ray, L. Samuels, and R. G. Parr, J. Chem. Phys. 70, 3680 (1979).

[11] J. L. Reed, J. Phys. Chem. 85, 148 (1981).

[12] R. T. Sanderson, Science 114, 670 (1951).

[13] R. W. Rudolph, W. L. Wilson, F. Parker, R. C. Taylor, and D. C. Young, J. Am. Chem. Soc. 100, 4629 (1978).

[14] R. W. Rudolph, W. L. Wilson, and R. C. Taylor, J. Am. Chem. Soc. 103, 2480 (1981).

[15] W. L. Wilson and R. W. Ruldolph, to be published.

[16] L. J. Bartolotti, S. R. Gadre, and R. G. Parr, J. Am. Chem. Soc. 102, 2945 (1980).

[17] K. D. Sen, P. C. Schmidt, and A. Weiss, Theoret. Chim. Acta (Berl.) 58, 69 (1980).

[18] J. D. Corbett and P. A. Edwards, J. Am. Chem. Soc. 99, 3313 (1977).

[19] C. H. E. Belin, J. D. Corbett, and A. Cisar, J. Am. Chem. Soc. 99, 7163 (1977).

[20] R. C. Burns, and J. D. Corbett, J. Am. Chem. Soc. 104, 2804 (1982).

[21] For reviews of ${ }^{119}$ Sn NMR data for compounds other than clusters see (a) P. J. Smith and L. Smith, Inorganica Chim. Acta Rev. 7, 11 (1973). (b) R. K. Harris, J. D. Kennedy, and W. McFarlane, in NMR and the Periodic Table, R. K. Harris and B. E. Mann, Eds. (Academic, New York/London, 1973), pp. 309-377.

[22] For a review see J. Mason, Adv. Inorg. Chem. Radiochem. 18, 197 (1976) and 22, 199 (1979).

[23] Y. Nomura, Y. Takeuchi, and N. Nakagawa, Tetrahedron Lett. 8, 639 (1969).

[24] A. A. Cheremisin and P. V. Schastnev, J. Mag. Resonance 40, 459 (1980).

[25] J. J. Burke and P. C. Lauterbur, J. Am. Chem. Soc. 83, 326 (1961).

[26] R. R. Sharp, J, Chem. Phys. 60, 1149 (1974). 